\title{
Synthesis, Degradation, Biocompatibility and Drug Release Studies of Bis 2-Hydroxy Ethyl Terephthalate-based Poly (Mannitol-Citric-Sebacate) Ester
}

\author{
Athira K S*, Sarkar K, Chatterjee K \\ Biomaterials and Tissue Engineering Laboratory, Department of Materials Engineering, \\ Indian Institute of Science, Bangalore-560012, India \\ * Corresponding author email: athiraiisc@ gmail.com
}

Received: 23 April 2016 / Revised: 05 June 2016 / Accepted: 16 June 2016 / Published: 20 June 2016

\section{ABSTRACT}

Bis 2-Hydroxy Ethyl Terephthalate-based biodegradable poly(mannitol-citric-sebacate) has been synthesized by catalyst-free melt condensation process using two different diacids and Bis 2-Hydroxy Ethyl Terephthalate with D-mannitol as monomers having a potential to be metabolized in vivo. The biocompatibility of the polymer, Bis 2-Hydroxy Ethyl Terephthalate-poly(mannitol-citric-sebacate) has been tested using human primary stromal cells. In vitro degradation of Bis 2-Hydroxy Ethyl Terephthalate-poly(mannitol-citric-sebacate) polymer in Phosphate Buffered Saline solution carried out at physiological conditions indicates that the degradation goes to completion after 23 days. The usage of Bis 2-Hydroxy Ethyl Terephthalate-poly(mannitol-citric-sebacate) polymer as a drug carrier has been analyzed by doping the polymer with Doxorubicin model drug and the release rate has been studied by mass loss over time. The cumulative drug-release profiles exhibit a biphasic release with an initial burst release and cumulative 100 percent release within 14 days.

Keywords: Bis 2-Hydroxy ethyl terephthalate poly(mannitol-citric-sebacate), Biodegradable Polymer, Human Primary Stromal Cells, Biocompatibility, Scaffold, Drug-delivery.

\section{Introduction}

Target release and sustained release could not be expected usually in conventional oral and intravenous drug administration [1]. Drugdelivery devices aid in the controlled release by providing therapeutic drug levels, restricting the drug-delivery to a specific site within the body and reducing the number of re-administrations during treatment [2]. Recently, the use of biodegradable polymers in the drug-delivery implants has gained much attention. The use of biodegradable polymer-drug implant systems for controlled release and target specificity in treating cancers and tumors has been studied and shown promising results [3]. Among the biodegradable polymers, aliphatic polyesters are used as drugdelivery carriers in local drug-delivery systems for in vivo applications due to their degradability and biocompatibility [4]. Some of the aliphatic polyesters have biodegradation times varies from weeks to years [5].

One of the most commonly used anticancer agents is 5-Fluorouracil (5-FU), but it is poorly absorbed after oral administration and thus local drug-delivery is the ideal way to overcome this disadvantages [6]. It is also one of the commonly used Doxorubicin (Dox) containing regimens and is available for clinical use in limited supply. Human primary stromal cells or mesenchymal stem cells produced by expanding bone marrow mononuclear cells in culture can be used for biocompatibility studies. The usage of these polymers in the biomedical field becomes common due to its degradation in physiological conditions inside the human body [7]. As temporary implants, they can stay intact until the healing process is complete; subsequently, they degrade and are excreted from the body as waste products [8]. 
Currently, typical commercially available chemosynthetic biomedical adhesives are cyanoacrylate adhesive (Dermabond R), aldehyde-based adhesive (GRF glue R) and fibrin-based adhesive (Bolheal R). Cyanoacrylate and aldehyde-based adhesives provide strong bonding, though cytotoxicity is relatively high. A fibrin-based adhesive is known to have a relatively low cytotoxicity but have an inferior bonding strength. Biodegradable adhesives were derived from citrate, malate and tartrate as organic acid-based crosslinkers. Excellent bonding strength, reactivity and bonding time were reported when using disuccinimidyl tartrate (DST) as a crosslinker and human serum albumin (HSA) as a hardener for the muscle of porcine, with the bonding strength being equivalent to that of cyanoacrylate-based adhesive [9]. Inoue et al. [10] artificially created to use the novel DSTHSA biological adhesive for repairing the longitudinal tear of the avascular zone of the meniscus, investigated the optimum usage and evaluated the effectiveness and safety using animal models. Many matrix types, which combine synthetic and natural materials, are able to enhance biocompatibility and biofunctionality and their blending into synthetic polymers improves the overall cytocompatibility of the scaffolds [11]. Inthanon et al. [12] reported on the design and fabrication of a synthetic scaffold, composed of a minimum of $90 \%$ sericin, a natural glue-like protein from Bombyx mori silkworm cocoons, and Poly(L-lactic-co- $\varepsilon$-caprolactone) as an effective substrate for the proliferation of undifferentiated Human Wharton's jelly mesenchymal stem cells, derived from umbilical cords and can be used in drug delivery and the controlled release of growth factor.

Biodegradable aliphatic polyesters are the preferred choices of materials for in vivo applications due to their degradability and biocompatibility [5]. Aliphatic polyesters have better degradation properties than aromatic esters as the aromaticity provides the hydrophobicity. Although many biodegradable materials, including polyesters, have been developed [13], most of them require stringent operating conditions, catalyst/initiators and multiple steps during synthesis and suffer from rigid mechanical properties and long degradation times [5]. It has been shown that poly (b-hydroxy octanoate) was not significantly degraded even after two years in an animal body [14]. The osmolarity and ion concentrations of Phosphate Buffered Saline (PBS) match with those of the human body (isotonic). PBS maintains a steady $\mathrm{pH}$ and acts as non-toxic to cells so that the cells are not destroyed.

\section{Materials and Methods}

\subsection{Synthesis of Bis 2-Hydroxyethyl Terephthalate-based Poly (mannitol-citric- sebacate) Ester}

Sebacic acid, Citric acid, D-Mannitol and Bis 2Hydroxyethyl terephthalate (BHET) have been purchased from S.D Fine Chemicals Ltd., India. BHET polyesters have been synthesized by melt condensation polymerization technique using the above monomers, as shown in Figure 1. In the glass tube kept on the stand; $6 \mathrm{~g}$ of Sebacic acid, $7.5 \mathrm{~g}$ of BHET and $2.85 \mathrm{~g}$ of citric acid have been taken. A magnetic bead has been put in the glass tube and inserted a needle with the rubber stopper to maintain it closed and kept the tube in the oil. Temperature has been set to $160^{\circ} \mathrm{C}$ on the heater to form a molten solution as the melting point of sebacic acid is around $133^{\circ} \mathrm{C}$, while that of BHET is around $110^{\circ} \mathrm{C}$ and that of citric acid is $153^{\circ} \mathrm{C} .1 .35 \mathrm{~g} \mathrm{D}$-Mannitol is added only after 1.5 hours due to the lower reactivity of BHET than D-Mannitol and the total polymerization took 2 hours, which is noted only after the liquefaction of the entire material.
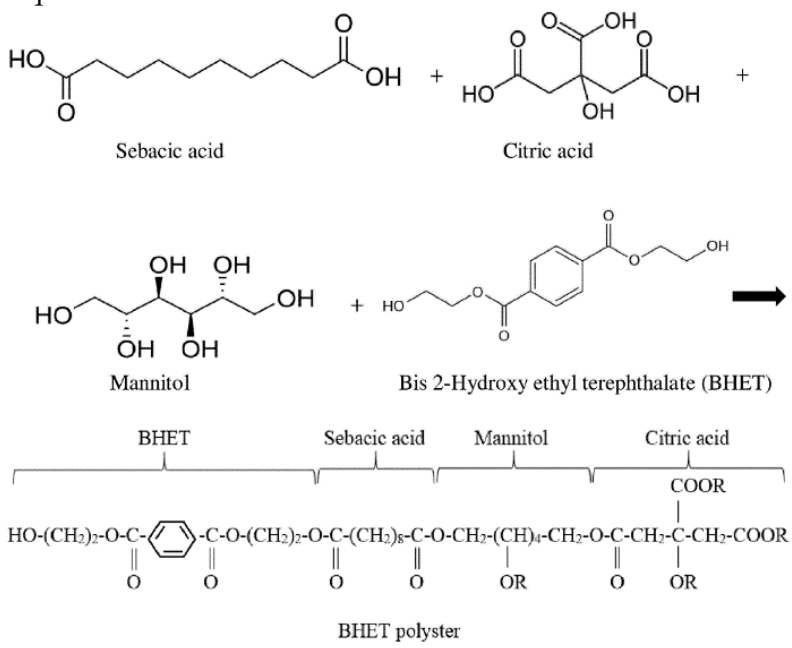

Figure 1: Synthetic scheme of the synthesis of BHET polyester 
After complete melting of solids, the solution is allowed to react for 1 hour with continuous stirring by setting rotation speed to $3 \mathrm{rpm}$. During the reaction, nitrogen gas is continuously purged through the glass tube to provide an inert atmosphere and aid the removal of water formed during the reaction. The prepolymer is taken directly for polymer-drug implant studies. For preparing the drug-loaded polymers, the prepolymer and drug have been dissolved in ethanol and subsequently, ethanol is evaporated. The prepolymers are post-polymerized in a vacuum oven for 2 days at $1000^{\circ} \mathrm{C}$ and $560 \mathrm{~mm}$ $\mathrm{Hg}$ to form cross-linked polyesters. The cured polymers have been used for drug-delivery, degradation and biocompatibility studies. $1 \mathrm{H}-$ NMR spectra for the polymer has been recorded on an NMR spectrometer at $400 \mathrm{MHz}$ using acetone as solvent and tetramethylsilane as the internal reference. The prepolymer has been purified by dissolving the polymers in $20 \mathrm{wt} \% 1$, 4-dioxane and precipitated in 3 vol $\%$ waterpropanol non-solvent mixture with continuous agitation, followed by filtration and freeze-drying at $-90^{\circ} \mathrm{C}$. The cured polymer is subjected to Fourier Transform Infra-Red (FTIR) analysis by scanning thin films made out of cured polymer and $\mathrm{KBr}$ crystals using FTIR spectrometer over the 4000-500 cm-1 range IR spectrum, at room temperature.

\subsection{Preparation of Phosphate Buffer Saline}

1x PBS, which corresponds to $1 \mathrm{ml}$ PBS is used and prepared as per Cold Spring Harbor Protocol [15]. $800 \mathrm{ml}$ of Milli-Q-water has been measured with a graduated cylinder and transferred to an Erlenmeyer flask. $8 \mathrm{~g}$ of $\mathrm{NaCl}, 0.2 \mathrm{~g} \mathrm{KCl}, 1.44 \mathrm{~g}$ of $\mathrm{Na}_{2} \mathrm{HPO}_{4}$ and $0.24 \mathrm{~g}$ of $\mathrm{KH}_{2} \mathrm{PO}_{4}$ are also transferred to the flask. 3 to 5 mins proper stirring is given allowing the solutes to dissolve so that oxygen is not introduced into the solution while it is rapidly mixed. it is ensured that there are no remaining particles of undissolved salts in the solution before adjusting the $\mathrm{pH}$. If particles are present, stirring is continued at reduced speed to get solution gently mixed. Properly calibration of the $\mathrm{pH}$ meter has been ensured and rinsed the $\mathrm{pH}$ probe with Milli-Q-water. The probe tip has been cleaned with a paper towel without touching it. $\mathrm{pH}$ probe is inserted into the solution and slowly added $1 \mathrm{M} \mathrm{HCl}$, drop wise with a transfer pipette and allowed the $\mathrm{HCl}$ to fully dissolve into the solution. Stopped stirring the solution and measured the $\mathrm{pH}$ with the $\mathrm{pH}$ meter. Repeated above steps until the $\mathrm{pH}$ of the solution become 7.4. The resultant solution has been poured into a freshly graduated cylinder and adjusted the final volume to 1 liter with Milli-Q-water.

\subsection{In vitro Degradation}

Phosphate buffer saline solution of $\mathrm{pH} 7.4$ has been prepared using monosodium diphosphate and disodium phosphate (S.D. Fine Chemicals Ltd., India) with fine adjustment of $\mathrm{pH}$ using diluted $\mathrm{NaOH}$. The square shaped polymer specimens of side $8-10 \mathrm{~mm}$ and $1.5-2 \mathrm{~mm}$ thickness has been immersed in $15 \mathrm{ml}$ of phosphate buffer solution and maintained at $370^{\circ} \mathrm{C}$ in a water bath with continuous agitation. At specified intervals of time, the polymer specimens have been taken out, washed with Milli-Q-water and dried to constant weight under vacuum. The percent degradation of the polymers in buffer saline solution has been calculated by mass loss over time.

\subsection{Scaffold Fabrication}

Porous scaffold from the polyester has been fabricated using the salt-leaching technique utilizing the solubility of the prepolymer in different solvents [16]. The prepolymer has been dissolved in 1, 4-dioxane separately to form 25 weight percent solution. Sieved salt is added to this solution to get a 25 percent w/v slurry. The slurry has been transferred to Teflon moulds and the solvent is allowed to evaporate slowly at $90^{\circ} \mathrm{C}$. After solvent evaporation for $24 \mathrm{~h}$, the moulds are transferred to a vacuum oven and the polymers are cured at $560 \mathrm{~mm} \mathrm{Hg}$ vacuum and $100^{\circ} \mathrm{C}$ for 3 days. The cured salt filled polymers have been demoulded and cut into square pieces of side $8-10 \mathrm{~mm}$. These samples are leached out of salt by incubating the polymer in Milli-Q-water and replacing the water medium every $12 \mathrm{~h}$ for 4 days. The resulting porous polymer has been freeze-dried at $-90^{\circ} \mathrm{C}$ and stored in desiccators. 


\subsection{Cell Culture}

The cytotoxicity test for evaluating the biocompatibility of a polymer in which the toxicity effect on the human cells due to the presence of the polymer or its degradation products is studied [17]. Initially, the polymer specimen of size, $10 \mathrm{~mm} \times 2 \mathrm{~mm}$ has been sterilized by rinsing with absolute ethanol and UV radiation for $30 \mathrm{mins}$. The source used for UV radiation is FS20 lamp with a Kodacel filter, emitting UVB spectrum of 280-320 nm wavelength at a lower dose. Human primary stromal cells or mesenchymal stem cells have been used in this study for evaluating the biocompatibility. These cells have been grown in high-glucose Dulbecco's Minimal Essential Medium (DMEM; Sigma) supplemented with Invitrogens such as 10 percent $(\mathrm{v} / \mathrm{v})$ fetal bovine serum, $100 \mu \mathrm{g} / \mathrm{ml}$ streptomycin and $100 \mu \mathrm{g} / \mathrm{ml}$ penicillin [18]. After growth, these cells have been harvested using trypsin 0.025 percent/EDTA 0.01 percent, and these cells have been resuspended by adding an equal volume of the medium. The suspended cells in the medium have been seeded in two polystyrene sterile tissue-culture dishes at a very low density of 1520 percent confluence, and $15 \mathrm{ml}$ of DMEM medium supplemented with FBS, streptomycin and penicillin are added. One petri dish served as the negative control and the other dish is loaded with sterilized polymer sample to study the cytotoxicity effect of the polymer. Both the disks are incubated at $37^{\circ} \mathrm{C}$ and 5 percent $\mathrm{CO}_{2}$, and the cells have been allowed to grow to form a confluent monolayer. The cells have been observed for growth in the dish through an inverted microscope every two days and the medium is replaced with a fresh medium on alternate days for a week.

\subsection{Drug-release Studies}

The required quantity of drug, Dox has been added to the polymer. Three bags have been made using a wire net. Three square pieces of the same measurements have been cut out from the polymer-drug matrix. Each bag is kept in the balance and after taring the balance, one piece of the polymer has been put into each bag and weighed. Put the bags with the polymer-drug matrix in PBS buffer with $15 \mathrm{ml}$ each and incubated it at $370^{\circ} \mathrm{C}$ under shaking. $1 \mathrm{ml}$ of the buffer is taken at specified intervals of time and added back $1 \mathrm{ml}$ of PBS buffer to the samples. Samples have been taken at different intervals of time. The absorbance of each of these samples is measured at $544 \mathrm{~nm}$ using a spectrophotometer till the absorbance reading comes to a standstill. The drug-polymer matrix samples have been analysed for drug-loading efficiency. Small cured polymer-drug matrix discs have been weighed and placed in $100 \mathrm{ml}$ of basic $\mathrm{NaOH}$ solution and allowed for complete dissolution of the polymer and the drug. The supernatant liquid has been analysed for the drug quantity using the absorbance level at the respective $\lambda \max$ for the drug, measured with a UV-Vis spectrophotometer. The percent degradation of the polymers in buffer saline solution has been calculated by comparing mass loss over time and graph is plotted.

\section{Results and Discussion}

\subsection{Polymer Synthesis}

Bis 2-Hydroxyethyl terephthalate-based poly(mannitol-citric-sebacate) ester; abbreviated as BHET-p(MCS) has been synthesized without the use of catalyst or initiators and hence expected of less or nil toxicity. On subsequent curing under controlled conditions, the reaction attains completion and forms a solid mass, which is highly cross-linked and not soluble in any solvent. Despite the small quantity of BHET, cross-linked structures are formed due to the trifunctionality of BHET and the polymer becomes cross-linked after curing. The NMR spectrum for this prepolymer is shown in figure 2 and has common peaks for many of its monomers. All the monomers are incorporated in the polymer because the mass loss during curing is less than $1.8 \%$. The peaks of Sebacic acid $\mathrm{CH} 2$ group and D-Mannitol $\mathrm{CH}$ and $\mathrm{CH} 2$ groups are merged with similar groups available in BHET. Thus, qualitative analysis can not use for determining the monomer ratio in the prepolymer. 
Athira et al., J. Mod. Mater.; Vol. 1, Issue 1, pp: 9-16, 2016

Table 1: BHET-p(MCS) degradation profile in PBS solution at $\mathrm{pH} 7.4$

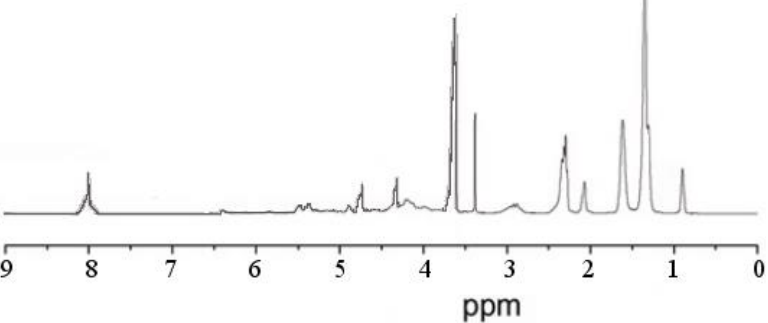

Figure 2: $1 H-N M R$ spectra of BHET-p(MCS) in acetone

FTIR spectrum for the synthesized BHET$\mathrm{p}(\mathrm{MCS})$ cured polymer is shown in figure 3 . The peaks for the specific functional groups in the IR spectrum such as Ester $\mathrm{C}=\mathrm{O}$ group stretching, double bond $\mathrm{C}=\mathrm{C}$ and the $\mathrm{C}-\mathrm{H}$ bending of end methyl group are identified and seen correspond to wave numbers $700-2930$ reciprocal centimetres.

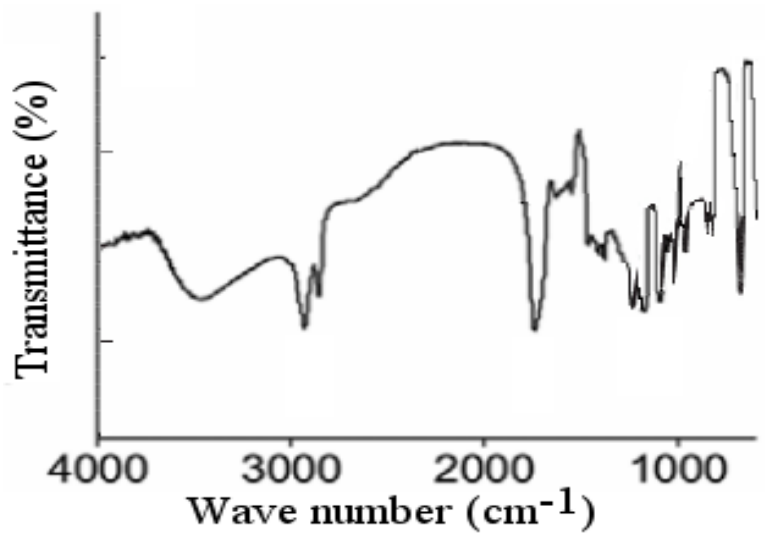

Figure 3: FTIR spectrum of Cured BHET-p(MCS) polymer

\subsection{In vitro Degradation}

Aliphatic, biodegradable polyesters start degrading when placed in an aqueous medium due to hydrolysis. The degradation behaviour of the polyester can be analyzed by the change in solution viscosity, molecular weight, as well as mass-loss measurement methods [19]. Since the synthesized cured polymer is cross-linked, the first two methods cannot be used and thus massloss measurements has been carried out to determine the degradation behaviour. The in vitro degradation of the polymer has been observed through the variation of the weight loss of the polymer with incubation time and the time for complete degradation of the polymer which is more than 23 days as given in table 1 .

\begin{tabular}{|l|c|c|c|c|c|}
\hline $\begin{array}{l}\text { Incubation time } \\
\text { (days) }\end{array}$ & 5 & 10 & 15 & 20 & 23 \\
\hline $\begin{array}{l}\text { Weight loss of } \\
\text { polymer (\%) }\end{array}$ & 44 & 58 & 67 & 75 & 78 \\
\hline
\end{tabular}

The degradation mechanism of the polymer in aqueous medium involved a combination of chemical reaction, diffusion and dissolution process. These include the following processes viz., water sorption; and reduction of modulus, strength, molar mass and weight. More hydrophilic backbone and end-groups; reactive hydrolytic groups in the backbone; porosity; less crystallinity and smaller size accelerate degradation mechanism. The mechanism begins with water diffusion followed by a stage in which oligomers with acidic end-groups autocatalyzed the hydrolysis reaction. A critical molecular weight is reached and oligomers start to diffuse out from the polymer. Water molecules diffuse into the void created by the removal of the oligomers which in turn encourages oligomer's diffusion. Marked decrease in polymer mass and a sharp increase in the oligomer diffusion rate occur as the oligomers diffuse from the porous regions. Polymeric matrix become highly porous and degradation proceeds homogeneously and becomes slower until complete degradation of the polymer.

\subsection{Scaffold Fabrication}

BHET-p(MCS) polymer has been fabricated as a porous sponge like scaffold by the salt leaching technique. The scaffold shows macro- and micropores between the polymer networks with the size of $18 \pm 12 \mu \mathrm{m}$ (Figure 4) for the latter. These interconnected micropores will serve as a passage route for the nutrients, deep inside the polymer scaffold, which is required for cell growth in tissue engineering [17]. The scaffold type of polymer network can be utilized in various vascular graft or tissue-engineering applications. For tissue-engineering applications, the excess hydroxyl or carboxyl groups available in the polymer backbone chain can be utilized as moieties for potential modification. Peptides or proteins can be incorporated into the polyester 
network using hydroxyl/carboxyl group functionalities to derive a particular cell response.

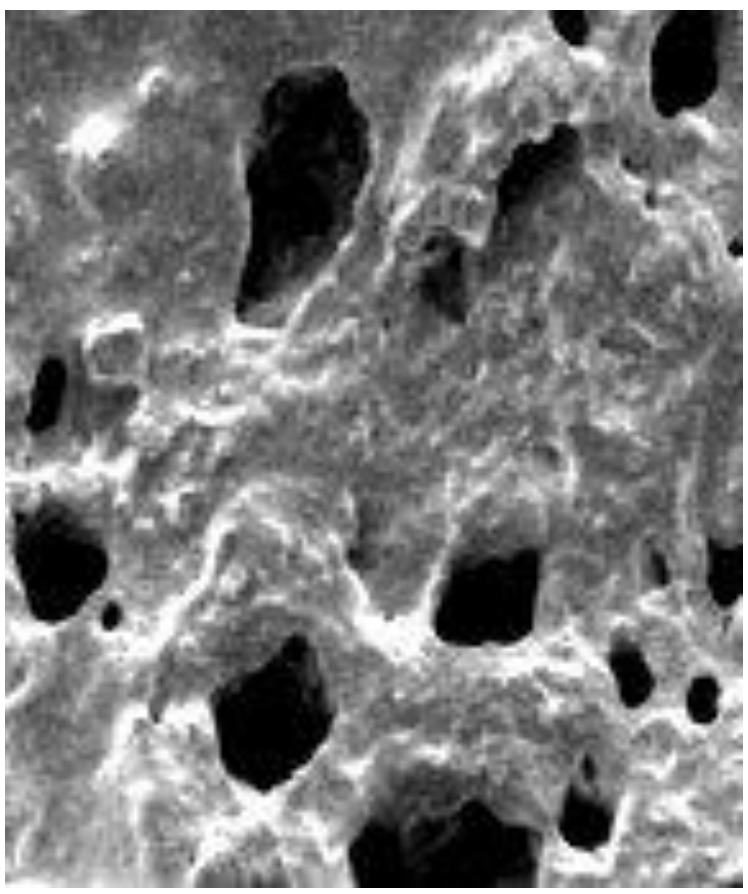

Figure 4: SEM image of BHET-p(MCS) polymer scaffold

\subsection{Cell Culture}

The performance of cells such as growth pattern and morphology in the presence of polymer has been compared with a negative control. The negative control in this experiment is a cell culture in polystyrene dish which is compatible with cells. It has been noticed on the second day of observation that the cells are attached at the bottom of both the dishes. The cell morphology is found to be similar in both the dishes and had a complete confluence in the dish as shown in figure 5. The morphology of the cells proves that they had survived in the presence of the polymer, were attached to the bottom of the dish and finally grew to occupy the whole area or full confluence. A broad range of colony sizes, representing varying growth rates and different cell morphologies, ranging from fibroblast-like spindle-shaped cells to large flat cells could be noticed. Cell debris or changes in morphology, such as cell lysis, loss of spindle shape or detachment from the bottom were not pronounced in the cell cultures with the presence of polymer. The results indicate that the polymer does not release any inhibitory leachate to the medium that is toxic and thus the polymer has been found to be non-cytotoxic.

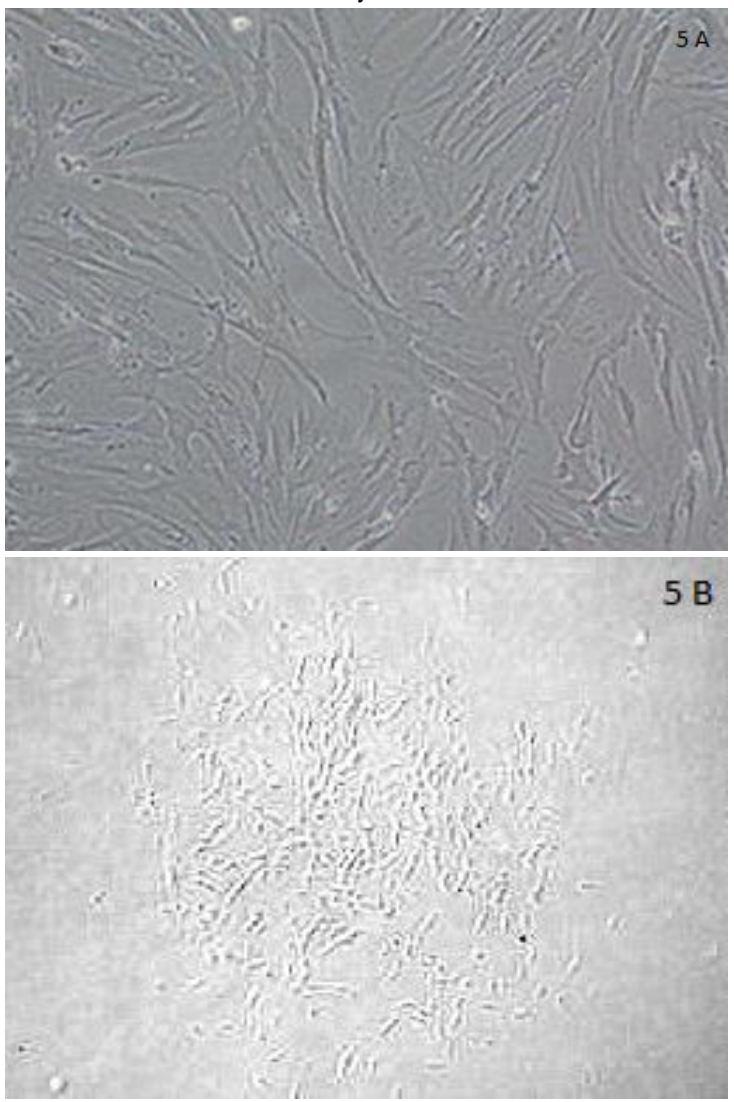

Figure 5: Morphology of Cell cultures

5 A: A Colony Forming Unit-Fibroblast after 7 days of culture (negative control),

5 B: Confluent cultures after 7 days in the presence of BHET-p(MCS) polymer

\subsection{Drug-delivery Studies}

Loading efficiency of the drug in the polymer has been found to be 89 percent. The dissolution of the drug in the medium and the diffusion of the medium through the matrix, swelling of the polymer and cross-linked density also play roles in manipulating the drug-release profiles [19]. Figure 6 shows the cumulative Dox drug release profile from BHET-p(MCS)-Dox implants for drug loadings in the polymer. Drug release from the polymer implant shows that the release profile shows biphasic expulsions with burst release in the initial phase and thereafter a constant release controlled by degradation and diffusion mechanism for Dox drug. The most desirable release profile from a polymer-drug implant is a constant release rate, but most of the drug implants have varying release rates. The initial burst release of the drug is mostly due to 
Athira et al., J. Mod. Mater.; Vol. 1, Issue 1, pp: 9-16, 2016

the fast dissolution of the drug from the implant surface, as there is no requirement of diffusion of medium inside the implant. This behaviour indicates the dispersion of the drug molecules well in the polymer matrix.

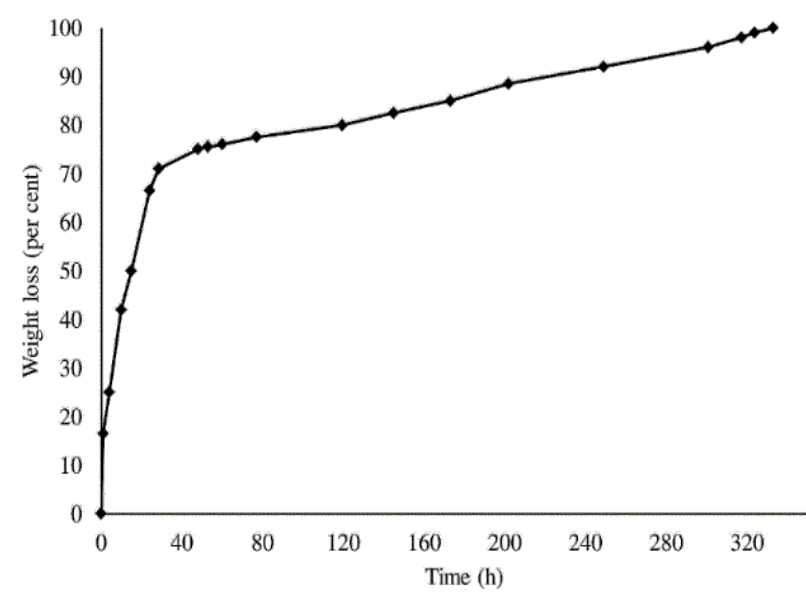

Figure 6: Percent drug release of Dox over time from BHET-p(MCS)

The 75 percent cumulative drug release from the implants takes place within $48 \mathrm{~h}$. The initial burst release could be due to rapid dissolution of drug molecules from the exposed surface. Similar type of initial burst behaviour has been observed with 5-FU drug from other polymer matrices and this characteristic of in vitro drug burst release is a common phenomenon in many drug-delivery systems [4]. The poly (carboxy phenoxy propane):sebacic acid matrix with Carmustine drug is a commercially available drug-delivery system known as 'Gliadel' and its drug-release profile also shows a similar initial burst behaviour [20]. The initial burst will be higher if it fills the surface more, whereas the burst release will be lower if there is also an even distribution of the drug inside the matrix. Thus the interaction of the polymer with the drug molecule plays a role in evenly distributing the drug molecule throughout the matrix. The distribution of Dox drug in the sample is not uniform throughout, with most of the drug available on the surface. On exposure to the PBS medium, the immediate dissolution of the drug in the medium also can be a reason for the rapid release. For cross-linked polymers, the initial hydrolysis changes the crosslink density of the polymer matrix, which does not necessarily involve weight loss. This results in an increasing diffusion of the medium inside the matrix and increased permeation rate of the drug caused by the gradually decreasing cross-linked density [6]. For higher drug loadings, the initial release rate of the drug is smaller, which eventually increases in due course of time as observed in other polymer systems with Dox drug [4]. Smaller drug release may be due to the comparatively higher distribution of the drug inside the matrix and the lower initial diffusion of water inside the matrix. Due to the creation of free space by drug expulsion, the latter rate for higher loading matrices are increased. Li et al. [19] reported that as the matrix releases the drug, more void pathways form inside the matrix, which increases the diffusion of medium inside and the drug solution outside resulting in increased release profile. The availability of the medium inside the matrix increases the hydrolysis reaction, which causes faster degradation.

\section{Conclusions}

BHET-p (MCS) polymers are synthesized from monomers without any catalyst and extreme operating conditions and found to be soft and flexible. The study with polymer-Dox implants shows that the drug release has a biphasic nature and a mass loss of 100 percent has been observed in 14 days for this polymer. The BHET-p(MCS) polymer has good biocompatibility for human stromal cells, found to be involved in human metabolism process, exhibit a fast degradation pattern in physiological conditions and can be used as drug implants for local delivery.

\section{Acknowledgements}

The authors are greatly indebted to Govt. of India for the KVPY fellowship granted to the first author for the research work included in this manuscript. The authors declare that there is no conflict of interest regarding the publication of this paper.

\section{How to Cite this Article:}

A. Sunil, S. K, and K. Chatterjee, "Synthesis, Degradation, Biocompatibility and Drug Release Studies of Bis 2-Hydroxy Ethyl Terephthalate-based Poly (Mannitol-Citric-Sebacate) Ester", J. Mod. Mater., vol. 1, no. 1, pp. 9-16, Jun. 2016. doi:10.21467/jmm.1.1.9-16 


\section{References}

[1] U. Edlund, and A. C. Albertsson, "Degradable polymer microspheres for controlled drug delivery," in Degradable Aliphatic Polyesters, vol. 157, A.C. Albertsson, Ed. Berlin: Springer, pp. 67-112, 2002.

[2] K. E. Uhrich, S. M. Cannizzaro, R. S. Langer, and K. M. Shakesheff, "Polymeric systems for controlled drug release," Chem. Rev., vol. 99, pp. 3181-3198, Nov., 1999.

[3] A. K., Azab, J., Kleinstern, V., Doviner, B., Orkin, M., Srebnik, A. Nissan, and A., Rubinstein, "Prevention of tumor recurrence and distant metastasis formation in a breast cancer mouse model by biodegradable implant of 131 Inorcholesterol," J. Control. Release, vol. 123, pp. 116-122, Aug., 2007.

[4] Z. J. Sun, C. Chen, M. Z. Sun, C. H. Ai, X. L. Lu, Y. F. Zheng, B. F. Yang, and D. L. Dong, "The application of poly (glycerol-sebacate) as biodegradable drug carrier," Biomaterials, vol. 30, pp. 5209-5214, June, 2009.

[5] P. A. Gunatillake, and R. Adhikari, "Biodegradable synthetic polymers for tissue engineering," Eur. Cells Mater., vol. 5, pp. 1-16, May, 2003.

[6] J. Heller, S. Y. Ng, and B. K. Fritzinger, "Use of poly (ortho esters) for the controlled release of 5-fluorouracyl and a LHRH analogue," J. Control. Release, vol. 6, pp. 217-224, Dec., 1987.

[7] U. Edlund, and A. C. Albertsson, "Polyesters based on diacid monomers," Adv. Drug Del. Rev., vol. 55, pp. 585609, April, 2003.

[8] L. Xue, and H. P. Greisler, "Biomaterials in the development and future of vascular grafts," J. Vasc. Surg., vol. 37, pp. 472-480, Feb., 2003.

[9] T. Taguchi, H. Saito, M. Iwasashi, M. Sakane, and N. Ochia, "Biodegradable adhesives composed of human serum albumin and organic acid-based crosslinkers with active ester groups," J. Bioact. Compat. Polym., vol. 24, pp. 546-559, Nov., 2009.

[10] T. Inoue, T. Taguchi, S. Imade, N. Kumahashi, and Y. Uchio, "Effectiveness and biocompatibility of a novel biological adhesive application for repair of meniscal tear on the avascular zone," Sci. Technol. Adv. Mater., vol. 13, pp. 064219-064224, Dec., 2012.

[11] J. Jin, S. I. Jeong, Y. M. Shin K. S. Lim, H. S. Shin, Y. M. Lee, H. C. Koh, and K. S. Kim, "Transplantation of mesenchymal stem cells within a poly(lactide-co- $\varepsilon$ caprolactone) scaffold improves cardiac function in a rat myocardial infarction model," Eur. J. Heart Fail., vol. 11, no. 2, pp. 147-153, Feb., 2009.

[12] K. Inthanon, D. Daranarong, P. Techaikool, W. Punyodom, V. Khaniyao, A. M. Bernstein, and W. Wongkham, "Biocompatibility Assessment of PLCLSericin Copolymer Membranes Using Wharton's Jelly Mesenchymal Stem Cells," Stem Cells Int., vol. 2016, pp. 1-16, 2016.

[13] Z. Y. Wang, Y. M. Zhao, F. Wang, and J. Wang, "Syntheses of poly (lactic acid-co-glycolic acid) serial biodegradable polymer materials via direct melt polycondensation and their characterization," J. Appl. Polym. Sci., vol. 99, pp. 244-252, Jan., 2006.

[14] Y. Marois, Z. Zhang, M. Vert, X. Deng, R. Lenz, and R. Guidoin, "Mechanism and rate of degradation of poly hydroxy octanoate films in aqueous media: a long-term in vitro study," J. Biomed. Mater. Res., vol. 49, pp. 216-224, Feb., 2000.

[15] M. R. Green, and J. Sambrook, "Molecular Cloning: A Laboratory Manual," 2nd ed., Cold Spring Harbor
Laboratory Press, Cold Spring Harbor, New York, vol. 3, appendix B.12, 1989.

[16] J. Yang, A. R. Webb, S. J. Pickerill, G. Hageman, and G. A. Ameer, "Synthesis and evaluation of poly(diol citrate) biodegradable elastomers," Biomaterials, vol. 27, pp. 1889-1898, March, 2006.

[17] D. S. Katti, S. Lakshmi, R. Langer, and C. T. Laurencin, "Toxicity, biodegradation and elimination of polyanhydrides," Adv. Drug Deliv. Rev., vol. 54, pp. 933961, Oct., 2002.

[18] J. P. Bruggeman, B. J. D. Bruin, C. J. Bettinger, and R. Langer, "Biodegradable poly (polyol sebacate) polymers," Biomaterials, vol. 29, pp. 4726-4735, Dec., 2008.

[19] X. Li, X. Deng, M. Yuan, C. Xiong, Z. Huang, Y. Zhang, and W. Jia, "In vitro degradation and release profiles of poly-DL lactide-poly (ethylene glycol) microspheres with entrapped proteins," J. Appl. Polym. Sci., vol. 78, pp. 140148, Oct., 2000.

[20] L. K. Fung, M. Shin, B. Tyler, H. Brem, and W. M. Saltzman, "Chemotherapeutic drugs released from polymers: distribution of 1, 3-bis(2-chloroethyl)-1nitrosourea in the rat brain," Pharm. Res., vol. 13, no. 5, pp. 671-682, May, 1996.

Publish your research article in AIJR journals$\checkmark$ Online Submission and Tracking

$\checkmark$ Peer-Reviewed

$\checkmark$ Rapid decision

$\checkmark$ Immediate Publication after acceptance

$\checkmark$ Articles freely available online

$\checkmark$ Retain full copyright of your article.

Submit your article at journals.aijr.in

Publish your books with AIJR publisher-

$\checkmark$ Publish with ISBN and DOI.

$\checkmark$ Publish Thesis/Dissertation as Monograph.

$\checkmark$ Publish Book Monograph.

$\checkmark$ Publish Edited Volume/ Book.

$\checkmark$ Publish Conference Proceedings

$\checkmark$ Retain full copyright of your books.

Submit your manuscript at books.aijr.org 